\title{
Monitoring of Quality Indicators in Pre Analytical Phase of Testing in the Clinical Biochemistry Laboratoryof A Tertiary Care Hospital Attached with Government Medical College.
}

\author{
Dr.Margit Gajjar ${ }^{1}$, Dr.Arpita Patel ${ }^{2}$, Dr.Shilpa Jain ${ }^{3}$ \\ ${ }^{I}$ Tutor, Department Of Biochemistry, Medical College, Baroda, Gujarat, India. \\ ${ }^{2}$ Assistant Professor, Department Of Biochemistry, Medical College, Baroda, Gujarat, India. \\ ${ }^{3}$ Professor: Department Of Biochemistry, Medical College, Baroda, Gujarat, India.
}

\begin{abstract}
Introduction: Quality indicators (QIs) are fundamental tools enabling users to quantify the quality of laboratory services. Pre-analytical errors account for more than $70 \%$ of the total number of laboratory errors.

Objective: To quantify performancein the pre analytical phase of testing in Clinical Biochemistry Laboratory, using quality indicators and compare our results with those in the literature to assess the quality of our laboratory services.

Methods:For preanalytical phase there are various QIs defined by International Federation of Clinical Chemistry (IFCC) and Laboratory Medicine Working Group on Laboratory Errors and Patient Safety (WGLEPS), among them we monitored four QIs. (1)Sample lost (QI-8) (2) Sample with inappropriate anticoagulant (QI-9) (3) Hemolyzed sample (QI-10) and (4)Sample with insufficient Quantity(QI-12).The data pertaining to these QIs was collected and QI scores were calculated between $1^{\text {st }}$ January to31 ${ }^{\text {st }}$ October, 2015. We then compared the QI scores with the quality specifications laid down in IFCC (WG-LEPS).We also calculated the Six sigma value for QIS.

Results: During the 10 months period, a total of 138262 samples were received in Clinical Biochemistry Laboratory. In this period 2012 preanalytical errors associated with these four QIs were recorded, accounting for $1.4 \%$ (2012/138262) of thetotal number of samples received. Among total preanalytical errors, $1.5 \%($ $31 / 2012)$ were $(Q I-8), 0.9 \%$ (19/2012) were (QI-9), $12.9 \%$ ( 261/2012) were (QI-10) and $84 \%$ (1701/2012) were (QI-12) When these results were compared with specifications of IFCC (WG-LEPS), QI-8, QI-9 and QI 10 were found to be within optimal level whereas QI-12 was within desirable range. Sigma value for (QI-8), (QI-9), (QI$10)$ and (QI-12)QIs were 5.0,5.2, 4.5 and 3.8respectively.

Conclusion: The preanalytical performance of our laboratoryis favorable and complies with international qualityspecifications.
\end{abstract}

Keywords:-pre analytical phase, quality indicators, six sigma metrics.

\section{Introduction}

Quality indicators (QIs) are fundamental tools enabling users to quantify the quality of laboratory services. ${ }^{1}$ QIsconstitute objective measures that can be used to evaluate critical health care dimensions (e.g. patient safety, effectiveness, equity, patient-centeredness, timeliness, and efficiency) ${ }^{2}$. QIs should be part of a coherent and integrated quality improvement strategy implemented according to the specifically developed International Standard for Medical Laboratories Accreditation (ISO 15189: 2012)which recognizes the need to subdivide the Total Testing Procedure (TTP) into pre-examination, examination and post-examination procedures, commonly defined as pre, intra and post-analytical phases. ${ }^{3}$

The 2012 ISO 15189 standard "Medical laboratories: Particularrequirements for quality and competence" establishesthat the preanalytical phase of the testing process beginswith the test request from the healthcare provider and includesthe requisition, preparation of the patient, collectionof the primary sample and transportation of the sampleto and within the laboratory. The preanalytical phase endswhen the analytical examination begins. Clause 4.12.4 of this standard, which used for medical laboratory accreditation,requires the implementation of QIs for systematically monitoring and evaluatingthe contribution of the laboratory to patient care and theidentification of improvement opportunities. ${ }^{4}$

According to recent evidence,pre and post-analytical steps have been found to be more vulnerable to the risk of error ${ }^{5}$.Pre-analytical errors account for more than $70 \%$ of the total number of laboratory errors so preanalytical phase of testing is an area of concern for laboratory services. ${ }^{6}$ 
The InternationalFederation of Clinical Chemistry(IFCC) and LaboratoryMedicine Working Group on Laboratory Errorsand Patient Safety (WG-LEPS) has made an importantcontribution to developing QIs for the preanalytical phase and specifications for those indicators. ${ }^{7-9}$ In a project focusedon reducing laboratory testing errors, the IFCC WG-LEPSdeveloped a series of QIsspecific to clinical laboratories.Of these, 16 are focused on the preanalytical phase (Table 1). ${ }^{9}$

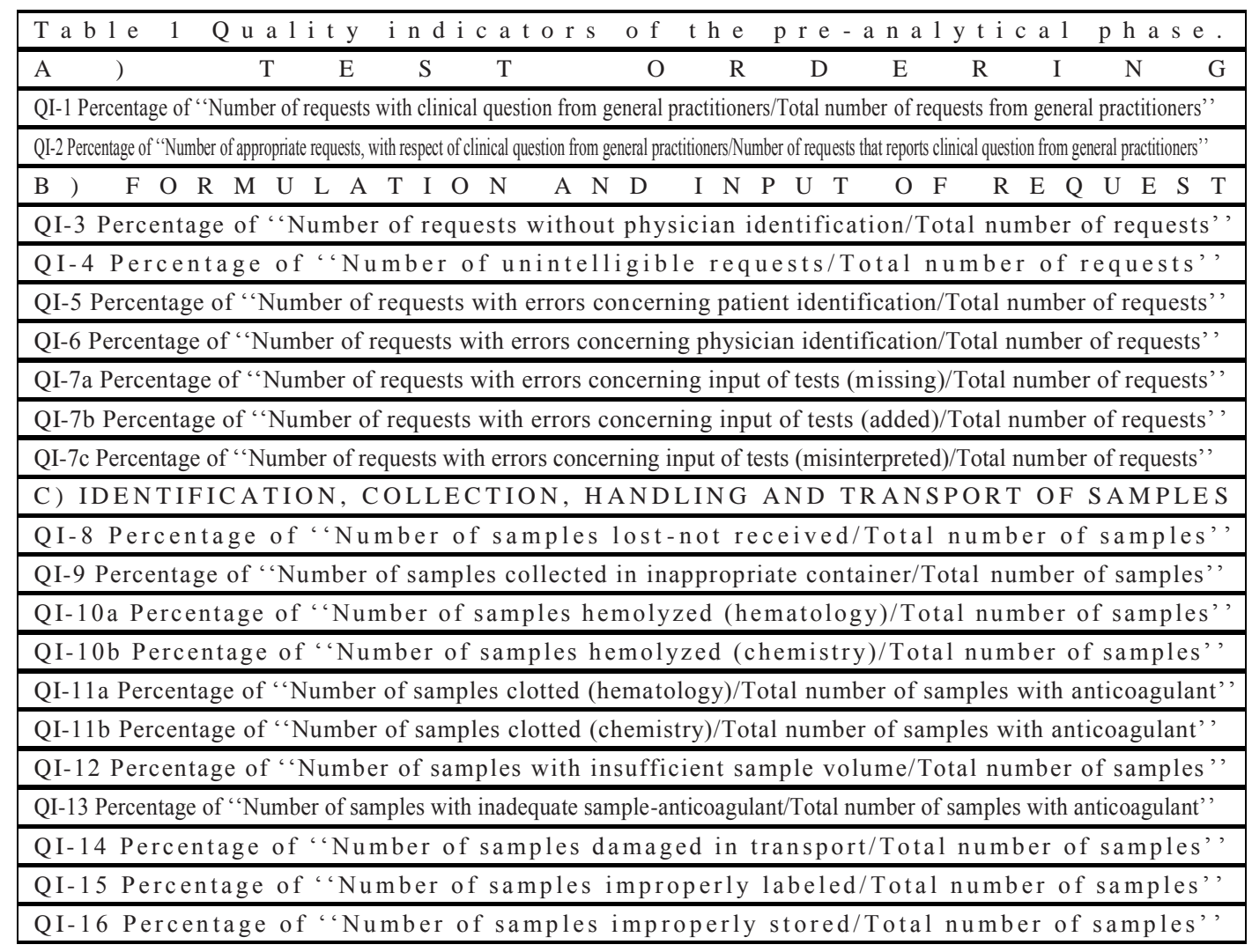

In the first stage of this project by IFCC WG-LEPS, thirty nine clinical laboratories were enrolled.Data were collected monthly for the period of 23 months (February 2008-December 2009). The data were analyzed and the following parameters were calculated for each QI (Table 2):

- the mean of results from each laboratory;

- the highest and lowest result, and the range obtained by participating laboratories;

- median and mean of results from all laboratories.

Three performance levels have been determined, minimum, desirable and optimum, defined in two ways depending on the distribution of results. When the range between the highest and lowest value was very wide, the median value was defined as the desirable level of performance. For those QIs specify in such a way that

- a higher score represented better performance e.g., QI-1 and QI-2, a value of greater than or equal to $25 \%$ above the median was defined as the optimum target, and a value less than or equal to $25 \%$ below the median was defined as the minimum target;

- a lower score represented better performance e.g., QI-3 to QI- 16, a value less than or equal to $25 \%$ below the median was defined as the optimum target, and a value greater than or equal to $25 \%$ above the median was defined as the minimum target.

In the case of uniformly distributed results and/or a narrow range, for those QIs specify in such a way that a higher score represented worse performance, the highest result was judged the minimum acceptable level of performance; the highest value divided by three the optimum performance level; and desirable performance was defined as the optimum level multiplied by two

The performance levels reported by the IFCC WG-LEPS forsome QIs for the preanalytical phase are show in Table 2. 
Monitoring Of Quality Indicators In Pre Analytical Phase Of Testing In The Clinical ...

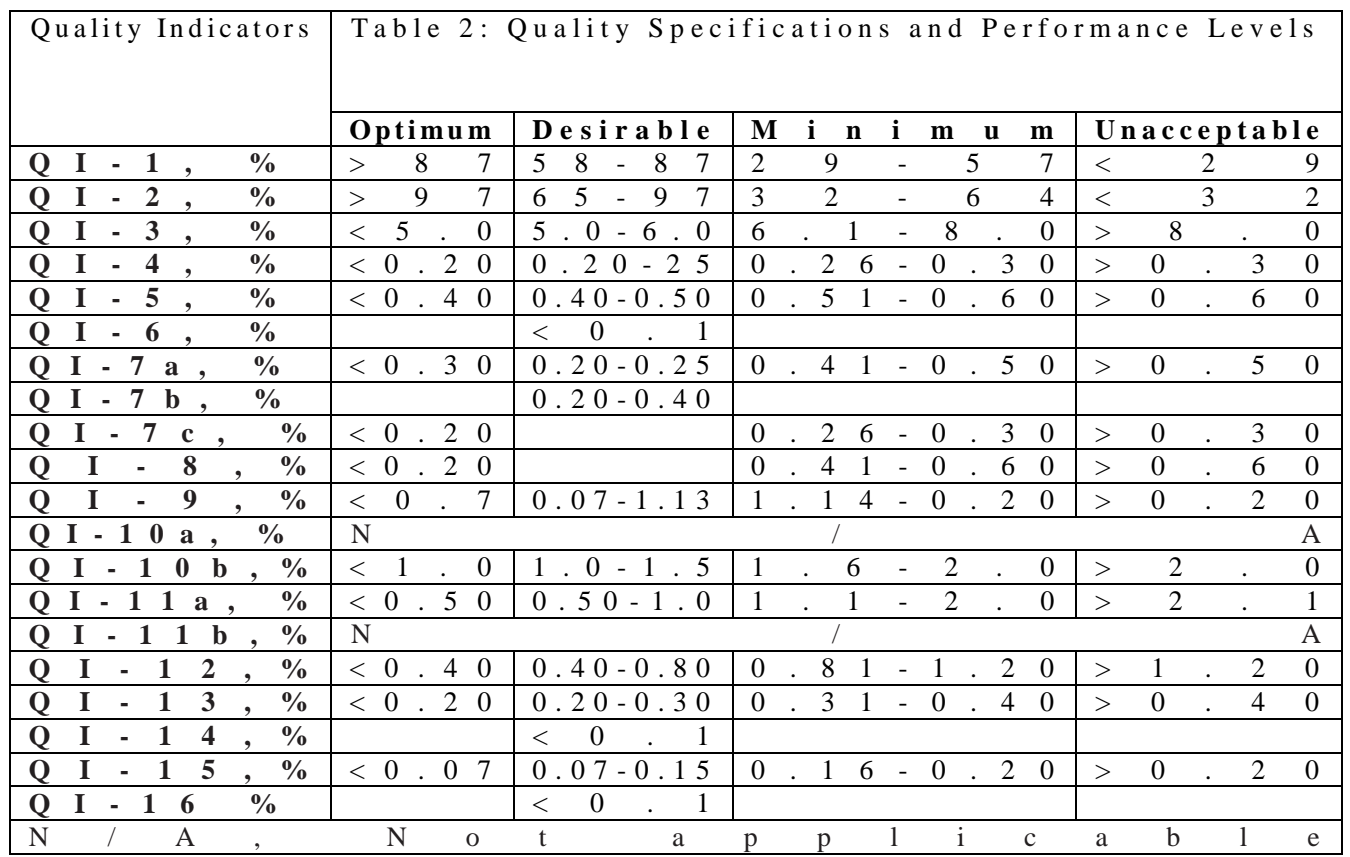

Another method of quality assessment, which is also applicablein the preanalytical phase, is the use of sigmametrics (i.e., the Six Sigma methodology). Six sigma metrics were developed byMotorola, Inc., this methodology was introduced intoindustry and business as early as the 1980s.Six Sigmaprovides principles and tools that can be applied to anyprocess to measure the defect and/or error rate. Bill Smith,known as the father of Six Sigma, decided tomeasure the defects per million (DPM) instead of defectsper thousand. The number of errors, or DPM, is a measureof laboratory performance ${ }^{10}$.The measurement of quality on asigma scale in the preanalytical phase requires monitoringof outcome process, counting the defects, calculatingthe DPM and using statistical tables to convert the DPMinto sigma metrics ${ }^{11}$. The sigma value indicates the frequency of errorsin a process. The higher this value, the less likely thelaboratory reports incorrect results. ${ }^{12}$ Quality is assessedon a sigma scale from 3 sigma as the minimum allowedfor routine performance to 6 sigma as best-in-class quality ${ }^{11}$ World-class quality processes have a six sigma level, which means around 3.4 errors per million. ${ }^{12}$ Average products,regardless of their complexity; have a quality performancevalue of approximately 4 sigma. ${ }^{13}$

\section{Organizational and ProceduralConditions for Data Collection}

We performed our study in the Clinical Chemistry Laboratory (NABL accreditated),Sir Sayajirao General Hospital (S.S.G.H.), Vadodara, which is major teaching hospital in Government setup in Eastern Gujarat. The laboratory performs emergency and routine tests for the patients attending the hospital, which has 1500 beds, with an annual average outdoor attendance of 4 lakh patients, an average annual indoor admission of 45000 patients and a bed occupancy rate of $83 \%$.It also offers 24 hours emergency services andvarious laboratories performs average 10,000 test parameters daily. All these high end facilities are given practically free of cost to the all patients. It is funded by the Department of the Health and Family welfare, Government of Gujarat.

Blood samples from inpatientsand the emergency department are collected bythe clinical ward staffwhereas outpatient samples are collected at Collection centre in the outpatient department (O.P.D.) by the laboratory staff. Venous blood samples are collected inplastic tubes with different additive as per the test requested. All laboratory tests are ordered viathe test request form. Request forms are assigned a unique colouridentification code. (Yellow for biochemistry, Pink for Hematology, White for serology and Green for microbiology).Urgent priority canbe specified by the provider on the request forms. Therequest form is duly filled, signed and stamped by the clinician andsent to the laboratory along with the samples. The specimens are transported by the ward staff(in specialized transport boxes to maintain the temperature) tothe laboratory reception area. The laboratory staffchecks whether the patient's identification data on thesample collection tube match those on the request form.

The laboratory has established acceptance and rejection criteria. In our laboratory, the sample rejection criteria are as follows;wrong, missing patient identification, wrong anticoagulant, too much or not enough sample volume and visible hemolysis. The samples that do not meet the acceptability criteria are rejected; data regarding these samples are recorded in a special register, and the staff members who collected them are 
notified. The date, a unique identification code, the reason for rejection and the name of the person who rejected the sample are specified in this register. Samples that meet the acceptability criteria are loggedin a register that specifies the time the samples werereceived and the number of tubes collected;all the samples are given specific laboratory identification number to categorize the samples accordingly. Subsequently, the samples are taken for centrifugation. After centrifugation, laboratory personnel visually check theblood samples to detect hemolyzed, lipemic and ictericserum. If hemolyzed, the concerned clinician is informed and sample details are recorded in Hemolyzed sample register. The laboratory personnel receiving the samples maintain this register.

Complying withthe ISO 15189:2012 standard that is implemented in the laboratory, the laboratory staff are trained to identify andregister all the errors that may affect the testing process, including those that occur in the pre-analytical phase. The collection centre staff and clinical staff have been trained to collect specimens. 'Primary sample collection manual', a handbook of instructions on proper techniques of all aspects of sample collection, has beendistributed toall wards and OPDs.

\section{Materials and Methods}

The aim of our study was to quantify performance in thepre-analytical phase of the testing process in Clinical Biochemistry Laboratory using quality indicators and to compare our resultswith those reported in the literature. We selected four QIs pertaining to the key activities of the pre-analytical phase. These were:

Samples lost-not received (QI-8);

Samples collected in a blood collection tube with inappropriate anticoagulant (other than the Clot activator vacuette or plain vacuette) (QI-9);

Hemolyzed samples (in biochemistry; QI-10); and samples with inadequate quantity (QI-12).

QI-9, 10, 12 were recorded from 'Sample rejection register' in the Clinical Chemistry Laboratory. Total number of samples being transported from various wards and OPDs, is maintained in the 'Sample transport register'. Totalnumber of samples received in the laboratory is matched with the number of the samples being transported. Deficient numbers of samples are considered as a sample lost (QI-8).

We calculated the sigma metric for these QIs. First, wecalculated the DPM rate using the following formula: $\mathrm{DPM}=($ number of errors $\times 1,000,000) /$ total numberof specimens or requests.

The DPM rate was converted to a sigma value based ontables available online (http://www.westgard.com/sixsigma-table.htm). For example, for the QI involving hemolyzedsamples, we calculated the sigma value as follows:

$\mathrm{DPM}=($ number of hemolyzed samples $\times 1,000,000) /$ total number of samples

In our study, the number of hemolyzed biochemistry sampleswas 261; the total number of samples was 138262 during the period from $1^{\text {st }}$ January to $31^{\text {st }}$ October, 2015. DPM is 4.5 .

Therefore,DPM = 261× 1,000,000)/ $138262=1888$.In the statistical tables, the sigma value for 1888

Sigma score calculators are also available at http://www.westgard.com/six-sigmacalculators-2.htm.

Daniela Stefania G. adopted four levels(similar to the WG-LEPS levels) of laboratory performance depending on the sigma values as given below ${ }^{14}$

1. Very good: $\geq 5$ sigma

2. Good: $4-<5$ sigma

3. Minimum: $3-<4$ sigma

4. Unacceptable: $<3$ sigma

These facilitate the identification of opportunities to improve laboratory services.

\section{Results}

During the 10 months period from $1^{\text {st }}$ January to $31^{\text {st }}$ October, 2015, a total of 138262 samples were received in the Clinical Chemistry Laboratory. 
Figure 1: Pre analytical QIs in Percentage

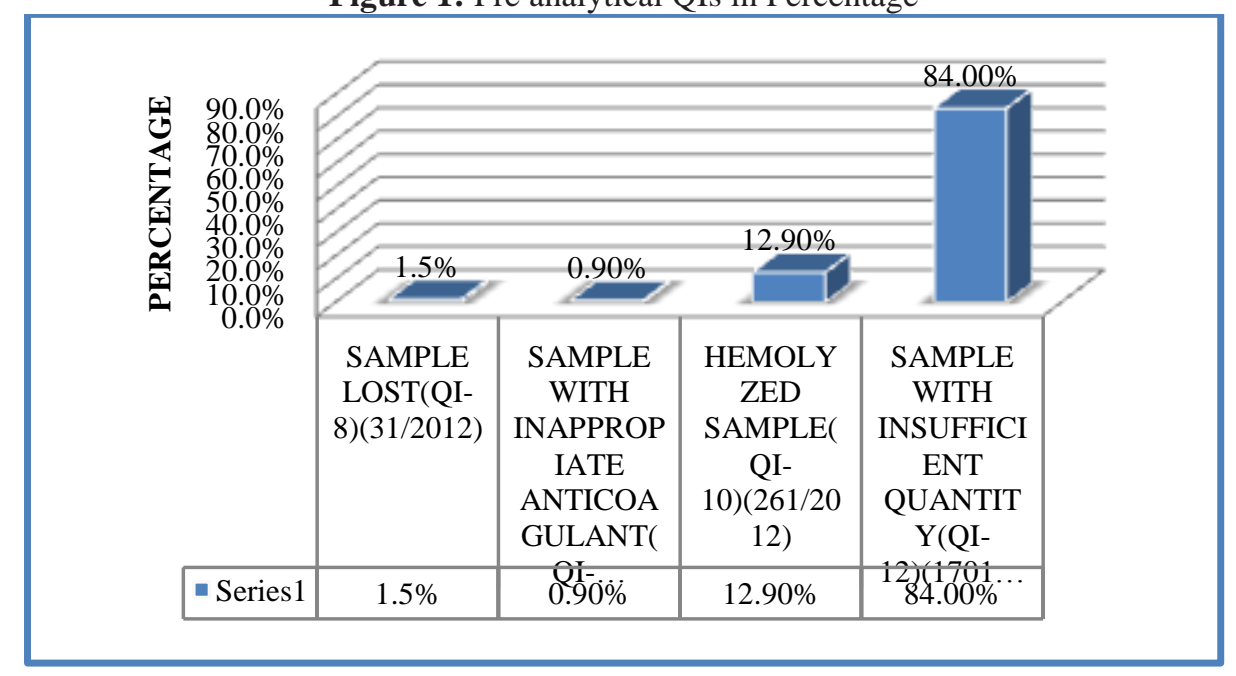

The total number of pre-analytical errors was 2012, whichaccounted for $1.4 \%$ of the total number of samples received duringthat period. As shown in the figure- 1, among the pre-analytical errors, $84 \%$ were quantity not sufficient samples $(\mathrm{QI}-12)($ sigma level $=3.8), 12.9 \%$ werehemolyzedsamples $(\mathrm{QI}-10)$ (sigma level $=4.5), 1.5 \%$ were samples notreceived in the laboratory $(\mathrm{QI}-8)($ sigma level $=5.0)$ and $0.9 \%$ samples with inappropriate container $(\mathrm{QI}-9)($ sigma level $=5.2)$

Table 3 shows the performance levels, based on IFCC and Sigma value of the QIs for pre analytical phase of testing in Clinical Chemistry Laboratory.

\begin{tabular}{|c|c|c|c|c|c|c|c|}
\hline $\begin{array}{l}\text { QI code and } \\
\text { meaning }\end{array}$ & Descriptor & $\begin{array}{l}\text { No. of } \\
\text { Errors }\end{array}$ & $\begin{array}{l}\text { Obtained } \\
\text { Value (\%) }\end{array}$ & $\begin{array}{l}\text { IFCC based } \\
\text { performance }^{\text {level }}\end{array}$ & DPM & $\begin{array}{l}\text { Sigma } \\
\text { Value }\end{array}$ & $\begin{array}{l}\text { Sigma based } \\
\text { Performance } \\
\text { level }^{\mathrm{B}}\end{array}$ \\
\hline $\begin{array}{l}(\mathrm{QI}-8) \\
\text { Samples lost- } \\
\text { not received }\end{array}$ & $\begin{array}{l}\text { Sample lost } / \text { Total } \\
\text { no. of samples }\end{array}$ & 31 & 0.02 & Optimal & 224 & 5.0 & Very Good \\
\hline $\begin{array}{l}(\text { QI-9) } \\
\text { Samples } \\
\text { collected in a } \\
\text { blood collection } \\
\text { tube with } \\
\text { inappropriate } \\
\text { anticoagulant } \\
\text { (other than the } \\
\text { Clot activator } \\
\text { vacuette or } \\
\text { plain vacuette) }\end{array}$ & $\begin{array}{l}\text { Samples collected in } \\
\text { a blood collection } \\
\text { tube with } \\
\text { inappropriate } \\
\text { anticoagulant (other } \\
\text { than the Clot } \\
\text { activator vacuette or } \\
\text { plain/ Total no. of } \\
\text { samples }\end{array}$ & 19 & 0.01 & Optimal & 137 & 5.2 & Very Good \\
\hline $\begin{array}{l}(\text { QI-10) } \\
\text { Hemolyzed } \\
\text { samples }\end{array}$ & $\begin{array}{l}\text { Hemolyzed samples/ } \\
\text { Total no. of samples }\end{array}$ & 261 & 0.18 & Optimal & 1888 & 4.5 & Good \\
\hline $\begin{array}{l}\text { (QI-12) } \\
\text { Samples with } \\
\text { inadequate } \\
\text { Quantity }\end{array}$ & $\begin{array}{l}\text { Samples with } \\
\text { inadequate Quantity/ } \\
\text { Total no. of samples }\end{array}$ & 1701 & 1.23 & Desirable & 12302 & 3.8 & Minimum \\
\hline
\end{tabular}

\section{Discussion}

Pre-analytical errors account for more than $70 \%$ of the total number of laboratory errors and have significant clinical and economic impacts on medical care. ${ }^{6} \mathrm{QIs}$ are useful performancemonitoring tools for the pre-analytical phase of thetesting process.

In our study, we selected four quality indicators. Other QIs can also beused; however, we did not examine these in the presentstudy. We recorded data on a daily basis regardingsamples that did not meet the acceptance 
criteria. Most of our results indicated an optimum level of performance; only one result (for QI-12) was just within thedesirable range, as per specifications laid by WG-LEPS.

\begin{tabular}{|l|l|l|l|l|}
\hline \multicolumn{5}{|c|}{ Table 4 Comparison of QI Performance level(\%) with other studies } \\
\hline QIs & Daniela at al ${ }^{14}$. & Chawla et al, ${ }^{15}$ & Lippi et al. ${ }^{16}$ & Present study \\
\hline $\begin{array}{l}\text { Sample lost } \\
\text { (QI- 8) }\end{array}$ & $0.05 \%$ & - & - & $0.02 \%$ \\
\hline $\begin{array}{l}\text { Sample with } \\
\text { inappropriate } \\
\text { anticoagulant } \\
\text { (QI-9) }\end{array}$ & $0.002 \%$ & - & - & $0.01 \%$ \\
\hline $\begin{array}{l}\text { Hemolyzed sample } \\
\text { (QI-10) }\end{array}$ & $0.4 \%$ & $0.7 \%$ & $0.77 \%$ & $0.18 \%$ \\
\hline $\begin{array}{l}\text { Quantity not sufficient } \\
\text { (QI 12) }\end{array}$ & - & - & - & $1.23 \%$ \\
\hline
\end{tabular}

Table 4 and 5 show comparison of QI performance level and sigma value with other studies. From the table it is evident that our QI-8, QI-9 and QI-10 scores are comparable to other studies. Performance of QI-8, 9 and 10 of our study is better whereas performance of QI-12 is lower compared with Sciacovelliet al ${ }^{17}$. Most of our results indicated an optimum level of performance; score of only one (for QI-12) was just within the desirable range and lower in sigma value, when compared with the specifications of the WG-LEPS.

The reason for more frequent errors in quantity of samples could be that in our institute, the personnel collecting samples change often. Ours being a teaching institute, new batch of interns and Post Graduate students are assigned the work of sample collection on rotational basis. They might not be able to learn the importance of proper quantity of samples in a short of period of their posting. The error of 'Inadequate quantity' mainly observed with Serum Electrolyte test, which required more amount of serum. Difficulty in sample collection of the pediatric patients is another major cause of insufficient quantity

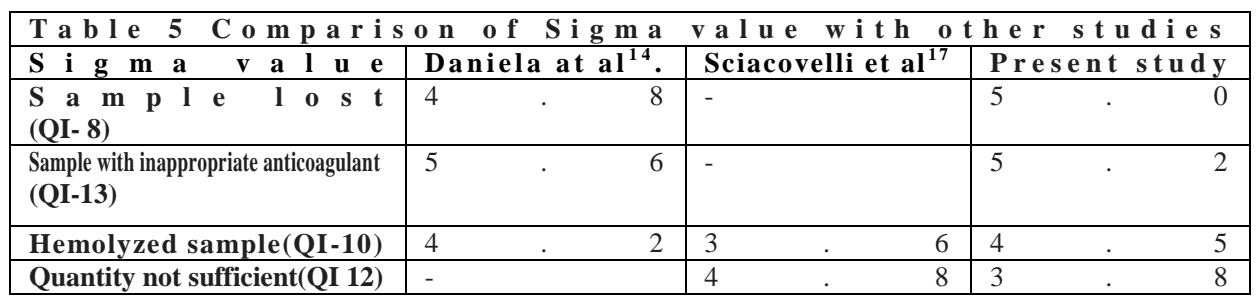

The reason for 'Sample lost-not received' was that sometimesafter registration for test, patient did not come to the collection centre or failed to come for postprandial sample. These errors were minor $(0.02 \%)$ and can be improved further by ensuring that patient has been instructed properly. Impressing upon the hospital staff assigned the work of transport of the samples. Importance of timely transport at proper location will also helps in minimizing the error of samplelost, (QI-8) (0.02\%) and samples collected in a blood-collection tube withinappropriate anticoagulant are minor $(0.01 \%)$, we consider themto be random errors.

Hemolyzed specimens for biochemical tests remain a challenge for clinicallaboratories. In our study, if any sign of hemolysis was detectedvisually, the sample was rejected and Clinicianwas asked to send new sample; It is usuallycaused by the use of small-gauge needles (smaller than21 gauge), excessive shaking or mixing of theblood sample after collection, centrifugation of the sampleat too high a speed for a prolonged period of time andcentrifugation of partially coagulated specimens.

\section{Conclusion}

In our study, none of the quality indicators we evaluated showed an unacceptable performance level in the pre analytical phase. Samplelost (QI-8),Sample with inappropriate container (QI-9) and Hemolyzed sample (QI-10) showed optimal level of performance and only one indicator Quantity not sufficient (QI-12) showed desirable level of performance. To minimize these errors, regular training, retraining and evaluation programmeare being organized for laboratory staff and induction training are being organized for newly posted interns and postgraduates.

Limitations of this study are, (1) we have not included all QIs. (2) In data collection, some errors might have been missed out from recording and (3) we have not traced the errors to various clinical ward/unit wise.

Further study can be doneto include monitoring of other QIs and to trace that which unit/ward have maximum number of rejection so that preventive and corrective actions can be taken. As continual improvement is 
necessary for the good laboratory practice, we continue to collect data regarding pre analytical errors to monitor this critical phase of laboratory testing to ensure ongoing satisfactory performance.

Funding:None declared

\section{Declarations}

Conflict of interest: Theauthor stat that there are no conflicts of interest regarding the publication of this article. Ethical approval:not required, as it was retrospective analysis of the data.

\section{References}

[1]. Plebani M. Quality indicators to detect pre-analytical errors in laboratory testing.ClinBiochem Rev. 2012;33:85-88.

[2]. Institute of Medicine Committee on Quality of Health Care in America.To Error is Human: Building a Safer Health System. Washington, DC: National Academies Press; 2000.

[3]. International Organization for Standardization. ISO 15189:2012:medical laboratories:particular requirements for quality and competence. Geneva, Switzerland: InternationalOrganization for Standardization; 2012.

[4]. International Organization for Standardization (ISO).ISO 15189:2007: Medical laboratories: particular requirements for quality andcompetence. Geneva, Switzerland: International Organization forStandardization; 2007

[5]. Plebani M, Carraro P. Mistakes in a stat laboratory: types and frequency. ClinChem1997;43:1348-51.

[6]. Carraro P, Plebani M. Mistakes in a stat laboratory: types and frequency 10 yearslater. ClinChem. 2007;53:1338-42.

[7]. Sciacovelli L, Plebani M. The IFCC working group on laboratoryerrors and patient safety.ClinChimActa. 2009;404:79-85.

[8]. Plebani M, Sciacovelli L, Lippi G. Quality indicators for laboratory diagnostics: consensus is needed. Ann ClinBiochem. 2011;48:479.

[9]. Sciacovelli L, O'Kane M, Skaik YA.Quality indicators in laboratory medicine: from theory to practice. Preliminary data from the IFCC Working Group Project "Laboratory Errors and PatientSafety”.ClinChem Lab Med. 2011;49:835-844.

[10]. Coskun A, Unsal I, Serteser M, Inal T. Six Sigma as a QualityManagement Tool: Evaluation of Performance in LaboratoryMedicine, Quality Management and Six Sigma.Rijeka, Croatia: InTechEurope;2010.

[11]. Westgard JO, Westgard SA. The quality of laboratory testing today.An assessment of $\sigma$ metrics for analytic quality using performance data from proficiency testing surveys and the CLIA criteria foracceptable performance.Am J ClinPathol. 2006; $125: 343-354$

[12]. Westgard JO, Klee GG. Quality management. In: Burtis CA,Ashwood ER, BrunsDE.editor. Tietz Textbook of Clinical Chemistry and Molecular Diagnostics.St.Louis,MO: Elsevier SaundersInc.;2006.

[13]. Nevalainen D, Berte L, Kraft C, Leigh E, Picaso L, Morgan T. Evaluating laboratory performance on quality indicators with the SixSigma scale. Arch Pathol Lab Med. 2000;124:516-519.

[14]. Daniela StefaniaG.,DAliborca Cristina viad.Quality indicators on preanalytical phase of testing in a stat Laboratory.La Medicine.2014;74-80

[15]. Chawla R, Goswami B, Singh B, Chawla A, Gupta VK, Mallika V.Evaluating laboratory performance with quality indicators. Lab Med.2010;41:297-300.

[16]. Lippi G, Bassi A, Brocco G, Montagnana M, Salvagno GL, GuidiGC. Preanalytic error tracking in a laboratory medicine department:results of a 1-year experience.Clin Chem. 2006;52:1442-1443.

[17]. Sciacovelli L, Sonntag O, Padoan A, Zambon CF, Carraro P, PlebaniM. Monitoring quality indicators in laboratory medicine does notautomatically result in quality improvement. Lab Med. 2011;50:463-469. 